\title{
A Survey of Classification of EEG signals using EMD and VMD for Epileptic Seizure Detection
}

\author{
Akshada Pradhan \\ Computer Science Engineering \\ Deaprtment \\ MIT-World Peace University \\ Pune, Maharashtra
}

\begin{abstract}
Automatic Classification of epileptic signals plays a critical role in long term monitoring and diagnosis. This review paper provides a survey of novel features of extraction and classification techniques for classifying epilepsy using Extreme Learning Machine (ELM) and Support Vector Machines (SVM) for Empirical Mode Decomposition (EMD) and Variational Mode Decomposition (VMD). The EEG signal is decomposed in Intrinsic Mode Functions (IMFs). Variance, Skewness and Kurtosis are drawn out as features from the decomposed signals. ELM and SVM are used as a classifier to classify EEG signals. For VMD, the EEG signal is decomposed to Intrinsic Mode Functions (IMFs) in the first place which are to be extracted as a feature after determining optimal features using classifiers like SVM and ELM.
\end{abstract}

Keywords_Epileptic, Electroencephalogram (EEG), Empirical Mode Decomposition (EMD), Variational Mode Decomposition (VMD), Support Vector Machines (SVM), Extreme Learning Machine (ELM)

\section{INTRODUCTION}

Translating thoughts and actions without acting physically has always been a staple of science fiction novels and Hollywood movies. However, this idea is fast becoming a reality: BCI is one such neurosciences advancement. The origins of BCI can be traced to work in the 1960s by Delgado (1969) and Fetz (1969). Delgado developed an implantable chip (which he called a "stimoceiver") that could be used to both stimulate the brain by radio and send electrical signals of brain activity by telemetry, allowing the subject to move about freely.[1] Though the stimoreceiver was used for entirely different purposes, BCI has emerged as a pivotal technology whose advancements in neuroscience have been of utmost importance to the medical community.

With the introduction of faster and cheaper computers, advances in our knowledge of how the brain functions, greater availability of devices for recording brain signals and more powerful signal processing and machine learning algorithms a sudden and lasting surge of interest and innovation can be seen in the field of BCI.

\section{BRAIN COMPUTER INTERFACE}

A brain-computer interface (BCI) is a technology that receives, analyses, and transfer the signals generated from brain into output commands in real world to accomplish a particular task.[2] In doing so, they are unique, as they do not include the normal neuromuscular pathways of peripheral nerves and muscles to perform a function, which is the site of pathology in paralyzed patients [3]. Computer-brain interfaces are designed to restore sensory function, transmit sensory information to the brain, or stimulate the brain through artificially generated electrical signals.

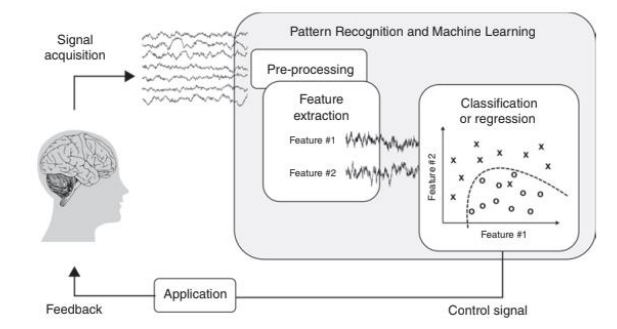

Fig. 1: Basic components of a brain-computer interface (BCI). (Image from Rao and Scherer, 2010).

One or more of the following processing stages are typically involved:

1. Brain recording: Signals from the brain are recorded using either invasive or noninvasive recording techniques.

2. Signal processing: Raw signals are pre-processed after acquisition (e.g., by bandpass filtering) and techniques for artifact reduction and feature extraction are used.

3. Pattern recognition and machine learning: This stage generates a control signal based on patterns in the input, typically using machine-learning techniques.

4. Sensory feedback: The control signal from the BCI causes a change in the environment (e.g., movement of a prosthetic arm or a wheelchair, change in the grip of a prosthetic hand), i.e. gives/produces an output. Some of these changes can be seen, heard, or felt by the user but in general, one can use sensors in the environment such as tactile sensors, force sensors, cameras, and microphones, and use the information from these sensors to provide direct feedback to the brain via stimulation. 5. Signal processing for stimulation: Before stimulating a particular brain region, it is important to synthesize an activity pattern for stimulation that mimics the type of activity normally seen in the brain region and that will have the desired effect/output.

6. Brain stimulation: The stimulation pattern received from the signal processing component mentioned in point 5 is used in conjunction with invasive or noninvasive stimulation techniques to stimulate the brain. 
It is clear from the stages of processing listed above that to begin building $\mathrm{BCIs}$, one must have a background in at least four essential areas: basic neuroscience, brain recording and stimulating technologies, elementary signal processing, and basic machine- learning techniques.[4]

As mentioned above, a basic understanding of neuroscience, recording \& stimulating technologies, elementary signal processing and ML techniques is required to understand BCIs.

$$
\text { III. SEIZURE TYPES }
$$

Seizure is simply the medical condition or neurological disorder in which too many neurons are excited at the same time caused by brain injury or by an imbalance of chemicals in the brain that is characterized predominantly by unpredictable interruptions of normal brain function. Epilepsy is another medical condition characterized by spontaneous recurrent seizures [1]. Epilepsy may lead to many injuries such as fractures, submersion, burns, motor vehicle accidents and even death. Approximate $1 \%$ of the total population develops epilepsy [2]. It is possible to prevent epileptic seizure with high sensitivity (i.e., detecting the preictal signal) if electrical changes in the brain that occur prior to the onset of an actual seizure can be detected.

The human brain processes sensory information received by external/internal stimuli. In the brain, neurons exploit chemical reactions to generate electricity to control different bodily actions and this ongoing electrical activity can be recorded graphically with an Electroencephalogram (EEG). EEG signals represent the nonlinear nature of the recorded signals in which there are two key terms, namely, 'state' and 'dynamics'. EEG signals from an epileptic patient can be divided into five periods or stages (i) nonseizure period- no epileptic syndrome is visible, (ii) ictal period-actual seizure period, normally duration is 1 to 3 minutes (iii) preictal period30 minutes to 60 minutes before ictal period, (iv) post-ictal period- 30 minutes after ictal period, and (v) interictal periodperiod between post-ictal period to pre-ictal period.

In order to gain crucial information if a seizure is going to occur or not is to analyse the preictal state of the EEG signals. With improving technologies and the increase in the number of quality channels, it is important to realize patterns that are potentially involved in EEG signals across a range of temporal scales [4].

\section{RECORDing AND STIMUlating THE BRAIN}

EEG is a technique used in measuring electrical signals produced by the brain that carry information about the functioning of the brain. These brain signals are recorded from the electrodes placed on the head, which is known as scalp EEG. Intracranial EEG signals, on the other hand, are recorded by placing needle electrodes into brain tissue.

EEG signals are mainly used to analyse brain conditions that have been conducted to explore sleep quality, emotion, attention and memory operation through the P300 signal, screening of depression, epilepsy detection and many more. There are five types of sub-bands of the EEG signal:

1. Delta Band (less than $4 \mathrm{~Hz}$ )

2. Theta Band $(4 \mathrm{~Hz}-8 \mathrm{~Hz})$

3. Alpha Band $(8 \mathrm{~Hz}-12 \mathrm{~Hz})$

4. Beta Band $(12 \mathrm{~Hz}-40 \mathrm{~Hz})$

\section{Gamma Band (greater than $40 \mathrm{~Hz}$ )}

The waveform varies according to the function of the brain's association with different tasks. For example, a recorded EEG signal during sleep has a higher percentage of long waves (delta and theta bands) whereas shorter waves (alpha and beta bands) dominate in the awake time.

In order to recognize patterns contained in EEG signals relevant features need to be extracted. These features may be time-domain features, frequency-domain features or both, where frequency content of the signals is revealed while maintaining the information of how the signals fluctuate through time.

The general process of classifying EEG signals is/paper is organized as follows:

1. Available Datasets

2. Feature Extraction

3. EEG signals transformation to another processing domain
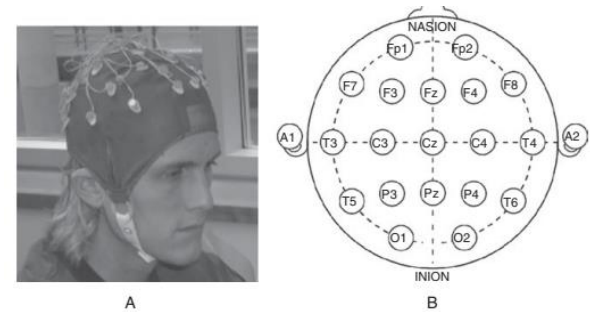

Fig. 2: Electroencephalography. (A) Subject wearing a 32 electrode EEG cap. (B) International 10-20 system for standardized EEG electrode locations on the head. $\mathrm{C}=$ central, $\mathrm{P}=$ parietal, $\mathrm{T}=$ =temporal, $\mathrm{F}=$ frontal, $\mathrm{F}_{\mathrm{p}}=$ frontal polar, $\mathrm{O}=$ occipital, $\mathrm{A}=$ mastoids (image $\mathrm{A}$ courtesy $\mathrm{K}$. Miller; image B from Wikimedia Commons).

\section{Classification}

V. AVALIABLE DATASETS FOR EEG SEIZURE CLASSIFICATION The EPILEPSIE dataset belongs to the European database developed in the EU-founded project "EPILEPSIE". So far, public resources for EEG recordings are limited. The EPILEPSIE database is by far the largest and most comprehensive database for human surface and intracranial EEG data. It is suitable for a broad range of applications e.g. of time series analyses of brain activity. The EU database contains annotated EEG datasets from more than 200 patients with epilepsy, 50 of them with intracranial recordings with up to 122 channels.

\section{FEATURE EXTRACTION}

Variance, Skewness and Kurtosis are taken as features from the decomposed signals of EMD. Variance $\left(\alpha^{2}\right)$, Skewness $\left(s_{k}\right)$ and Kurtosis $\left(\mathrm{k}_{\mathrm{u}}\right)$ are respectively given by: 


$$
\begin{aligned}
& \sigma^{2}=\frac{1}{N} \sum_{i=1}^{N}\left(x_{i}-\mu\right)^{2} \\
& \mathrm{Sk}=\frac{1}{N} \sum_{i=1}^{N}\left(\frac{x_{i}-\mu}{\sigma}\right)^{3} \\
& \mathrm{Ku}=\frac{1}{N} \sum_{i=1}^{N}\left(\frac{x_{i}-\mu}{\sigma}\right)^{4}
\end{aligned}
$$

Where $\mu$ is the mean and N is the length of the IMF, $\sigma$ is the standard deviation and $\mathrm{x}_{\mathrm{i}}$ is the IMF of the signal for the ith sample. Variance, Skewness and Kurtosis describe the dispersion, asymmetry and peakness of the dataset.

\section{EMPIRICAL MODE DECOMPOSITION}

EMD stands for Empirical Mode Decomposition. This method analyses nonstationary signals and has been popularly used for analysing biological signals for lung sound identification, electrocardiogram, seizure type detection and artefact removal.

This method works by decomposing a signal $s(t)$ into a number of band limited functions known as Intrinsic Mode Functions (IMFs) and residual by removing the local oscillation of the signals. These IMFs should satisfy two basic conditions:

1. The number of extrema or zero crossings must be the same or differ at most by one.

2. At any point, the average value of the envelope defined by local maxima and the envelope defined by local minima is zero.

IMFs are mono-component signals. Mathematically, the first IMF is subtracted from the original data

$$
\rho(\tau)=\xi(\tau)-\delta(\tau)
$$

This procedure is then applied iteratively to the residue $\mathrm{r}(\mathrm{t})$ until it becomes constant or contains no more oscillations.

\section{VARIATIONAL MODE DECOMPOSITION}

VMD is known as Variational Mode Decomposition approach for time frequency decomposition of EEG signals. This approach is used to overcome limitations in EMD approach.

EMD uses a recursive approach which does not allow backward correction and hence is unable to handle noise properly. In VMD, on the other hand, instead of a recursive approach, a concurrent approach is used to extract IMFs from the signal where the centre frequencies are dynamically calculated.

Here, an adaptive method is used, wherein the signal is decomposed into $\mathrm{k}$ IMFs and which gives a set of modes $u_{k}$ with their respective centre frequency wk. The sum of these modes represents the original signal.

In VMD the identification of IMFs is considered as an optimization problem. The VMD algorithm can be briefly summarized as follows:
1. For each mode $u_{k}$, the associated analytic signal is computed using Hilbert transform in order to obtain a unilateral frequency spectrum.

2. Mode's frequency spectrum is shifted by mixing with an exponential to the respective calculated centre frequency.

3. Bandwidth is estimated through Gaussian smoothness of the demodulated signal.

\section{ClassifIERS}

The effectiveness of the parameters (area and mean frequency of IMFs) in classifying the seizure and seizure-free EEG signals is evaluated using

1. LS-SVM: Support Vector Machines are supervised learning models that are a potential methodology for solving problems in linear and nonlinear classifications, function estimations and kernel-based learning methods. However, SVMs have a drawback: they offer a high computational burden of the constrained optimization problem. Hence to overcome these drawbacks, LS-SVM, known as least squares SVM, an extended version of SVM is used. It learns nonlinear mappings from the training set features. For a two-class SVM, we consider the following equation:

$$
\phi(\xi)=\sigma \imath \gamma \nu\left[\omega^{\mathrm{T}} \gamma(\xi)+\beta\right]
$$

Where $\mathrm{w}$ is the 1 dimensional weight vector, $\mathrm{b}$ is the bias and $\mathrm{g}(\mathrm{x})$ is the mapping function that maps $\mathrm{x}$ into the 1 dimensional space.

2. ELM: ELMs are feed-forward neural networks for classification, regression, clustering, sparse approximation, compression and feature learning with a single layer of hidden nodes, where the parameters of hidden nodes need not to be tuned.

Single feed-forward neural network (SFLN) possessing L-hidden node with additive and Radial Basis Function (RBF) is given by

$$
\mathfrak{R}_{\varphi} \gamma\left(\omega_{\varphi}, \beta_{\varphi}, \xi_{\mathrm{l}}\right)=\mathrm{O}_{\varphi}
$$

ELM is carried out in three steps:

a. Assigning randomly input weights $\mathrm{w}$ and bias $b$

b. Calculating the hidden layer output matrix $\mathrm{H}$. Determining the output weights $\beta^{\wedge}=\mathrm{H}^{-1} \mathrm{~T}$ where $\mathrm{H}^{-1}$ is the Morre Penrose generalized inverse of $\mathrm{H}$.

\section{CONCLUSION}

In a nutshell, $\mathrm{BCI}$ is an innovative technology that uses brain signals to control an external device to accomplish a task bypassing normal neuromuscular pathways. BCI has been used in medical applications like seizure detection, sensory rotations, motor rotations and rehabilitation. Among these epilepsy detections has been heavily researched. This review paper attempts to present epilepsy detection through EEG 
signals. EMD and VMD techniques are proposed to reduce input signals into respective IMFs. These signals then undergo feature extraction to select appropriate features to classify. Classifiers like SVM and ELM (Extreme Learning Machine) have been applied. The aforementioned methods can be advantageous in classification of biological signals such as Alzheimer and MS disease.

\section{ACKNOWLEDGMENT}

I thank Dr. Sharmishta Desai, Assistant Professor at MITWorld Peace University, Pune for assistance with guiding me with sufficient knowledge to write this paper and for comments that greatly improved the manuscript. I am immensely grateful for her comments on the earlier versions of the manuscripts, although any errors are my own and should not tarnish the reputation of this esteemed person.

\section{REFERENCES}

[1] Guido Domhege, Jose del R. Milan, Thilo Hinterberger, Dennis J. McFarland, Klaus-Robert Muller, "Toward Brain-Computer Interfacing: The MIT Press(2007)"

[2] Shiv Kumar Mudgal, Suresh K Sharma, Jitendra Chaturvedi, Anil Sharma, "Brain computer interface advancement in neurosciences: Applications and issues"

[3] I. Lazarou, S. Nikolopoulos, P. C.Petrantonakis, I. Kompatsiaris, M. Tsolaki, "EEG based brain-computer interfaces for communication and rehabilitation of people with motor impairment: a novel approach of the 21 st Century"

[4] Rao R.P.N., "Brain Computer Interfacing: An Introduction- Cambridge University Press(2013)" 